\title{
Effect of continuous psoas compartment block and intravenous patient controlled analgesia on postoperative pain control after total knee arthroplasty
}

\author{
Jae Jin Lee, Sang Sik Choi, Mi Kyoung Lee, Byung Gun Lim, and Wonseok Hur \\ Department of Anesthesiology and Pain Medicine, Korea University Guro Hospital, Seoul, Korea
}

Background: Total knee arthroplasty (TKA) generates severe postoperative pain in $60 \%$ of patients and moderate pain in $30 \%$ of patients. Because inadequate postoperative pain control can hinder early physiotherapy and rehabilitation, it is the most influential factor dictating a good outcome. The purpose of this study was to evaluate the effectiveness of continuous psoas compartment block (PCB) in comparison to intravenous patient-controlled analgesia (IVPCA) in TKA patients.

Methods: 40 TKA patients were randomly divided into 2 groups. Group IVPCA $(\mathrm{n}=20)$ received intravenous patient controlled analgesia (IVPCA) for 48 hours. Group PCB $(n=20)$ received continuous PCB for 48 hours at the fourth intertransverse process of the lumbar using the C-arm. Pain scores, side effects, satisfaction, the length of hospital stay, rescue antiemetics, and analgesics were recorded.

Results: Pain scores (VNRS 0-100) were higher in Group IVPCA than in Group PCB. Nausea and sedation occurred more frequently in Group IVPCA than in Group PCB. There were no differences between the groups in the length of the hospital stay, satisfaction scores, and the use of rescue antiemetics and analgesics.

Conclusions: Continuous PCB seemed to be an appropriate and reliable technique for TKA patients, because it provided better analgesia and fewer side effects such as nausea and sedation when compared to IVPCA. (Korean J Anesthesiol 2012; 62: 47-51)

Key Words: Lumbar plexus, Nerve block, Patient controlled analgesia, Postoperative pain, Total knee arthroplasty.

Received: June 3, 2011. Revised: 1st, July 11, 2011; 2nd, August 7, 2011; 3rd, August 8, 2011; 4th, August 8, 2011. Accepted: August 9, 2011. Corresponding author: Sang Sik Choi, M.D., Ph.D., Department of Anesthesiology and Pain Medicine, Korea University Guro Hospital, 80, Guro-dong, Guro-gu, Seoul 152-703, Korea. Tel: 82-2-2626-1870, Fax: 82-2-851-9897, E-mail: clonidine@empal.com (c) This is an open-access article distributed under the terms of the Creative Commons Attribution Non-Commercial License (http:// creativecommons.org/licenses/by-nc/3.0/), which permits unrestricted non-commercial use, distribution, and reproduction in any medium, provided the original work is properly cited. 


\section{Introduction}

With the growth of the aging population and regressive disorders, the need for total knee arthroplasty (TKA) has grown. However, its postoperative pain is severe and $60 \%$ of the patients complain of severe pain and $30 \%$ complain of moderate pain [1]. The postoperative pain of TKA prevents the use of physical therapy and rehabilitation therapy, which is intensive in the early period. This ultimately results in a bad prognosis [2]. Therefore, many studies have attempted to design method to reduce the postoperative pain of TKA. The commonly used intravenous patient controlled analgesia (IVPCA) is easy to set up, but a large dose of opioids is used to control the pain at the local surgical site or a small dose of opioids is used due to the anxiety of side-effects from overuse. However, these approaches led to cases of failure in pain control [3-5]. On the other hand, continuous nerve blocks have been shown to decrease pain, produce fewer side-effects, and decrease the lengths of hospital stays and rehabilitation when compared to IVPCA $[3,6]$. The continuous psoas compartment block (PCB), which unilaterally affects a vast area of the lower extremity, is one of the continuous nerve blocks. In this study, continuous PCB was compared to IVPCA with the goal of evaluating their effectiveness in postoperative pain control and their side-effects in TKA patients.

\section{Materials and Methods}

The study was performed on 40 patients who were below the age of 80, American Society of Anesthesiologists (ASA) class I and II, and were undergoing TKA. After the present study received the permission from the Institutional Review Board, the authors told the patients and guardians the purpose of the study, explained to them the possible side-effects, and got their informed consent. The patients were randomly grouped as follows: patients with IVPCA were placed into Group IVPCA $(n=20)$, patients who got a PCB by approaching the area of the $3^{\text {rd }}$ lumbar nerve were put into Group PCB $(n=20)$. Patients who had allergies to the drugs used in the study such as local anesthetics, a severe liver or kidney disorder, contraindications to having a peripheral nerve block (infection, coagulation disorder, or a disorder of the nervous system affecting the lower extremities), or a BMI of $30+$ were excluded from the study.

All the patients underwent general anesthesia. In Group IVPCA, $100 \mu \mathrm{g}$ of fentanyl, $0.5 \mathrm{mg}$ of hydromorphone, 15 mg per $10 \mathrm{~kg}$ body weight of keromin and normal saline in a total volume of $45 \mathrm{ml}$ were used. Continuous intravenous administration was applied at a $0.5 \mathrm{ml} / \mathrm{hr}$ rate using patientcontrolled analgesia [Ambix anaplus ${ }^{\circledR}$, E-Wha Fresenius Kabi, Korea]. The IVPCA was set so that the patient could have an infusion at each occurrence of pain with a $0.5 \mathrm{ml}$ bolus dose and a 15 min lockout time. The IVPCA was maintained until 48 hours after the surgery.

The continuous PCB was performed before the surgery. The patients were put into the prone position. Using a C-arm [Exposcop 8000, Ziehm imaging GMBH, Germany], the location of the $4^{\text {th }}$ lumbar vertebra body was confirmed, and $1 / 3$ of the outer area of the transverse process on the same side of the knee receiving surgery was set as the puncture site. At the puncture site, the skin wheel was made using local anesthetics. An epidural needle [Tuohy 18G, Perifix ${ }^{\circledR}$, B.Braun, Germany] was then inserted vertically into the skin and when the needle touched the transverse process, it was slightly taken out and was advanced parallel to the spine in the direction of the head at a degree of 10-20. At this point, the loss of resistance was confirmed using the air syringe. Afterwards, when the spreading of the contrast agent [Iopamiro ${ }^{\circledR} 300$, Bracco, Italy] around the psoas compartment was confirmed (Fig. 1), a catheter [20 G, Perifix ${ }^{\circledR}$, B.Braun, Germany] was inserted $2-3 \mathrm{~cm}$ deeper via a needle, and then the needle was removed. To prevent the movement of the catheter and reduce the risk of infection, subcutaneous tunneling was performed. $0.25 \%$ ropivacaine at a bolus dose of $20 \mathrm{ml}$ was infused via the catheter before the surgery. The nerve block was then confirmed using a pinprick test and a cold sense loss. After the surgery, continuous PCB boxter [AutoFuser ${ }^{\circledR}$, Acemedical, Korea] using $0.2 \%$ ropivacaine at a rate of $8 \mathrm{ml} / \mathrm{hr}$ was infused while leaving the catheter in place for 48 hours after the surgery.

Pain scores were measured using verbal numeric rating scales (VNRS) with a 0 to 100 range at $6 \mathrm{hrs}, 24 \mathrm{hrs}$, and $48 \mathrm{hrs}$ after surgery. During the hospital stay the patients were checked

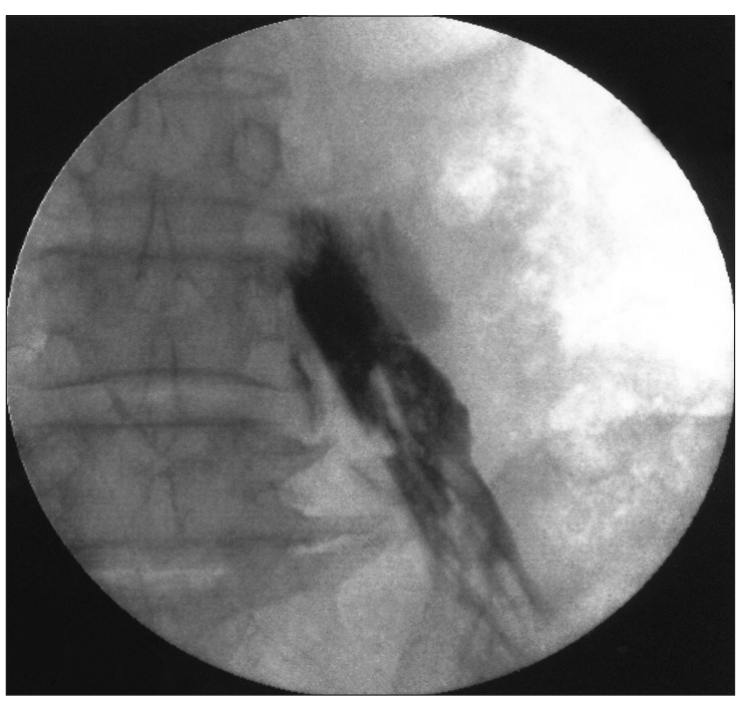

Fig. 1. C-arm images showing that the dye (Iopamiro ${ }^{\circledR} 300$ ) spread over the psoas compartment shadow. 
for postoperative side-effects. Nausea $(0=$ no nausea; $1=$ mild; $2=$ moderate; $3=$ severe $)$ of at least 1 and sedation $(0=$ alert; 1 = drowsy; 2 = sleeping, easy to arouse; 3 = sleeping, difficult to arouse) of at least 2 were included. At the time of patient discharge, the patient was directly surveyed for their satisfaction scores $(0=$ very unsatisfied, $100=$ very satisfied $)$. The length of the hospital stay, additional use of rescue antiemetics and analgesics were recorded. With severe nausea and vomiting, 10 mg of metoclopramide was administered and if the VNRS was more than $40 \mathrm{~mm}, 90 \mathrm{mg}$ of diclofenac was administered. The administration of the two drugs was recorded as well.

Using a VNRS of $20 \mathrm{~mm}$ as the basis, significance level $(\alpha)$ as 0.05 , and the power as 0.8 , the sample size was set at 19 patients per group. When considering the drop rate, the number of patients per group was set at 20. Statistical analysis was conducted using SPSS for Window version 12. 0, and all the quantative data was analyzed using ANOVA. Side-effects were analyzed using the Chi-square test. For post-hoc testing, Tukey and Dunnett's T3 were used. Quantative data was shown as mean $\pm \mathrm{SD}$, and the remaining was shown as numbers. A P

Table 1. Demographic and Perioperative Data

\begin{tabular}{lcc}
\hline & IVPCA $(\mathrm{n}=20)$ & PCB $(\mathrm{n}=20)$ \\
\hline Sex (M/F) & $4 / 16$ & $4 / 16$ \\
Age (yr) & $63.9 \pm 8.8$ & $66.1 \pm 7.8$ \\
Height (cm) & $155.6 \pm 6.9$ & $156.4 \pm 7.3$ \\
Weight (kg) & $64.9 \pm 8.3$ & $65.5 \pm 9.9$ \\
BMI & $26.7 \pm 2.7$ & $26.1 \pm 3.0$ \\
ASA physical status I/II & $5 / 15$ & $4 / 16$ \\
Ane. time (Min) & $161.2 \pm 17.2$ & $162.2 \pm 15.0$ \\
\hline
\end{tabular}

All values except sex and ASA physical status are expressed as mean \pm SD. Sex and ASA physical status are expressed as the number of patients. BMI: body mass index. ASA: American Society of Anesthesiologists. Ane. time: anesthetic time.

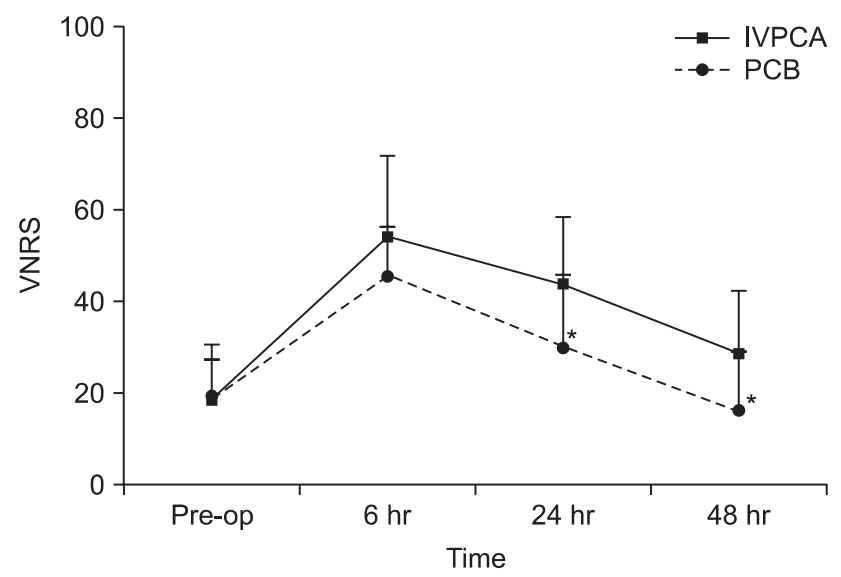

Fig. 2. A comparison of pain scores according to groups using a verbal numeric rating scale (VNRS) for postoperative pain in Group IVPCA and Group PCB according to time. ${ }^{*} \mathrm{P}<0.05$ compared to Group IVPCA. value $<0.05$ was considered statistically significant.

\section{Results}

There were no statistical differences between the two groups in regards to age, gender, BMI, ASA class, and anesthetic time (Table 1). There were no statistical differences between Group PCB (45.2) and Group IVPCA (54.0) in postoperative pain scores 6 hours after the surgery; however, 24 hours and 48 hours after the surgery, the postoperative pain scores were significantly lower in Group PCB $(29.5,16.0)$ than Group IVPCA $(43.5,28.5)$ (Fig. 2).

None of the patients experienced serious complications such as hypoventilation, nerve damage or hemorrhaging at the site of PCB. There were significantly more frequent incidences of nausea and sedation in Group IVPCA than in Group PCB. There were no statistical differences in vomiting or voiding difficulty (Table 2).

There were no statistical differences in satisfaction scores or the length of the hospital stay between the two groups. Rescue antiemetics and sedatives were used more frequently in Group IVPCA than in Group PCB, but no statistical differences were observed (Table 3 ).

\section{Discussion}

Postoperative pain of TKA causes the reflexive constriction of the quadratus femoris muscle, which results in increased pain and damage to the muscle function. These effects hinder

Table 2. Incidences of Side Effects According to Groups

\begin{tabular}{lcc}
\hline & IVPCA $(\mathrm{n}=20)$ & PCB $(\mathrm{n}=20)$ \\
\hline Nausea & $10(50)$ & $4(20)^{*}$ \\
Vomiting & $5(25)$ & $2(10)$ \\
Voiding difficlty & $2(10)$ & $2(10)$ \\
Sedation & $7(35)$ & $1(5)^{*}$ \\
Respiratory depression & $0(0)$ & $0(0)$ \\
Hematoma & - & $0(0)$ \\
Nerve injury & - & $0(0)$ \\
\hline
\end{tabular}

Values are expressed as the number (\%) of patients. $* \mathrm{P}<0.05$ compared to Group IVPCA.

Table 3. Comparison of the Length of the Hospital Stay, Satisfaction Score, Antiemetics and Analgesics According to Groups

\begin{tabular}{lcc}
\hline & IVPCA $(\mathrm{n}=20)$ & PCB $(\mathrm{n}=20)$ \\
\hline Length of hospital stay (days) & $17.7 \pm 5.1$ & $17.3 \pm 4.0$ \\
Satisfaction score (0-100) & $74 \pm 14$ & $81 \pm 12$ \\
Rescue antiemetics & $7(35)$ & $3(15)$ \\
Rescue analgesics & $14(70)$ & $10(50)$ \\
\hline
\end{tabular}

The length of hospital stay and the satisfaction scores are expressed as mean \pm SD. Rescue antiemetics and analgesics are expressed as the number of patients (\%). 
early walking and rehabilitation, which are important to a quick recovery and early hospital discharge $[2,7,8]$. Therefore, postoperative pain management is considered an important prognosis factor. Methods to manage postoperative pain of TKA are IVPCA and regional anesthesia. Continuous PCB, continuous epidural nerve block, and continuous femoral nerve block are examples of regional anesthesia.

Continuous PCB wets the psoas compartment with local anesthetics and blocks the nerve which passes through it. The psoas compartment is the area between the psoas muscle and the quadratus lumborum muscle. This area is innervated by the rami of the lumbar plexus and sacral plexus, and it consists of the genitofemoral nerve (L1-2), lateral femoral cutaneous nerve (L2-3), femoral nerve (L2-4), obturator nerve (L2-4), and partially the sciatic nerve (L4-S3) [9]. Therefore, blocking this area with a PCB is known to have better effects than not only IVPCA but also a continuous femoral nerve block, which usually blocks the obturator nerve that originates from the posterior branch [10-13]. Another regional anesthetic method, the continuous epidural nerve block, is a bilateral nerve block that limits the movement of the patient, so one should consider the risk of voiding difficulty and rare but serious complications such as epidural hematoma [14-16]. Therefore, this procedure cannot be easily performed on patients with a coagulation disorder or under the administration of anticoagulants to prevent deep venous thrombosis or pulmonary embolism. But the PCB only blocks the nerve unilaterally, and it can be performed even when there is a coagulation disorder. It is difficult to block of the obturator nerve with a continuous femoral nerve block. Thus, it is known that a continuous PCB is more effective in pain management than a continuous femoral nerve block, which has the disadvantage of requiring a separate block for the obturator nerve $[11,17]$.

In a continuous PCB, the sciatic nerve cannot be easily blocked, so there may be difficulties in complete pain control of postTKA. On the other hand, performing a post-TKA continuous PCB together with a sciatic nerve block has been reported to be as effective as a continuous epidural nerve block for pain control [18]. The presented study would have had better results if a sciatic nerve block was performed as well. However, each nerve block requires a separate catheter, resulting in further discomfort of inserting two catheters and the need to manage them. Therefore, the authors only performed a continuous PCB without performing a sciatic nerve block.

Watson et al. [19] and Raimer et al. [18] reported that continuous PCB also resulted in lower pain scores and fewer incidences of side effects such as nausea and sedation when compared to IVPCA. In the present study, the postoperative pain scores 6 hours after surgery between Group IVPCA and Group PCB were not very different. But afterwards, Group PCB had significantly lower pain scores than Group IVPCA. This is believed to have occurred because the psoas compartment has to be full with an adequate dose of local anesthetics to have an effect during continuous PCB. However, in the present study, the anesthetics were infused at a rate of $8 \mathrm{ml} / \mathrm{hr}$, because of this, the effect was low until 6 hours had passed. After the area was adequately loaded with local anesthetics, the pain scores in Group PCB were lower than in Group IVPCA. Also, peripheral nerve blocks like continuous PCBs increased tissue perfusion with local anesthetics and broke the vicious cycle of paintetany-pain due to the effects of muscle relaxation, as argued by Morin et al. [20].

IVPCA, which uses a large quantity of opioids, can be accompanied by nausea, sedation, and hypoventilation. However, only local anesthetics are used to block a local region, so the occurrences of systemic side-effects in intravenous patient controlled analgesia were low. In continuous PCBs, complications such as nerve damage, postoperative hemorrhage, durg infusion of the epidural, the subarachnoid space and the blood vessels can occur. Chayen et al. [21] conducted a PCB with 100 patients, and they reported 1 case of intravascular drug infusion and 1 case of epidural puncture. Although it may be safely said that PCB produces relatively low side-effects, the authors aimed at reducing such fatal complications by performing a continuous PCB with the additional use of C-arm images. After inserting the catheter, the authors confirmed that the contrast agent spread in the psoas compartment area before infusing the drugs. Since postoperative hemorrhaging, nerve damage, complications such as epidural and intravascular drug infusion did not occur in the present study, the authors found that it was better to have a continuous PCB using a C-arm than performing this procedure blindly. Group IVPCA had higher usage rates of rescue antiemetics and analgesics than Group PCB because of difficult pain management, nausea, and vomiting but the differences were not statistically significant. This is believed to be the case because the number of patients in the study was low and only the administrations of antiemetics and analgesics were recorded without considering their total doses.

In conclusion, the authors found that continuous PCB was good for controlling postoperative pain in TKA patients. This procedure was able to manage pain more effectively than IVPCA, and it produced lower systemic side-effects such as neausa and sedation. Thus, this method was deemed to be a good procedure that can be safely and effectively performed.

\section{References}

1. Chung MY, Kim CJ. The effect of bilateral femoral nerve block combined with intravenous patient-controlled analgesia after a bilateral total knee replacement. Korean J Pain 2008; 21: 211-6. 
2. Singelyn FJ, Deyaert M, Joris D, Pendeville E, Gouverneur JM. Effects of intravenous patient-controlled analgesia with morphine, continuous epidural analgesia, and continuous three-in-one block on postoperative pain and knee rehabilitation after unilateral total knee arthroplasty. Anesth Analg 1998; 87: 88-92.

3. Capdevila X, Barthelet Y, Biboulet P, Ryckwaert Y, Rubenovitch J, d'Athis F. Effects of perioperative analgesic technique on the surgical outcome and duration of rehabilitation after major knee surgery. Anesthesiology 1999; 91: 8-15.

4. Ganapathy S, Wasserman RA, Watson JT, Bennett J, Armstrong KP, Stockall CA, et al. Modified continuous femoral three-in-one block for postoperative pain after total knee arthroplasty. Anesth Analg 1999; 89: 1197-202.

5. Kim YJ, Kim YJ, Kim DY. Effect of Single-injection Femoral Nerve Block Using Real-time Ultrasound on the Postoperative Pain after Bilateral Total Knee Arthroplasty: $0.25 \%$ vs $0.5 \%$ Levobupivacaine Korean J Pain 2009; 22: 234-40.

6. Chelly JE, Greger J, Gebhard R, Coupe K, Clyburn TA, Buckle R, et al. Continuous femoral blocks improve recovery and outcome of patients undergoing total knee arthroplasty. J Arthroplasty 2001; 16: 436-45.

7. Schultz P, Anker-Møller E, Dahl JB, Christensen EF, Spangsberg N, Faunø P. Postoperative pain treatment after open knee surgery: continuous lumbar plexus block with bupivacaine versus epidural morphine. Reg Anesth 1991; 16: 34-7.

8. Horasanli E, Gamli M, Pala Y, Erol M, Sahin F, Dikmen B. A comparison of epidural anesthesia and lumbar plexus-sciatic nerve blocks for knee surgery. Clinics (Sao Paulo) 2010; 65: 29-34.

9. Hogan MV, Grant RE, Lee L Jr. Analgesia for total hip and knee arthroplasty: a review of lumbar plexus, femoral, and sciatic nerve blocks. Am J Orthop (Belle Mead NJ) 2009; 38: E129-33.

10. Atim A, Ergin A, Kurt E, Ozdemiroglu Y, Guzeldemir E. Comparison of sciatic psoas compartment block and sciatic femoral 3-in-1 block for knee arthroscopy. J Clin Anesth 2007; 19: 591-5.

11. Capdevila X, Macaire P, Dadure C, Choquet O, Biboulet P, Ryckwaert $\mathrm{Y}$, et al. Continuous psoas compartment block for postoperative analgesia after total hip arthroplasty: new landmarks, technical guidelines, and clinical evaluation. Anesth Analg 2002; 94: 1606-13.
12. Marhofer P, Nasel C, Sitzwohl C, Kapral S. Magnetic resonance imaging of the distribution of local anesthetic during the three-inone block. Anesth Analg 2000; 90: 119-24.

13. McNamee DA, Parks L, Milligan KR. Post-operative analgesia following total knee replacement: an evaluation of the addition of an obturator nerve block to combined femoral and sciatic nerve block. Acta Anaesthesiol Scand 2002; 46: 95-9.

14. Ben-David B, Schmalenberger K, Chelly JE. Analgesia after total knee arthroplasty: is continuous sciatic blockade needed in addition to continuous femoral blockade? Anesth Analg 2004; 98 : 747-9.

15. Campbell A, McCormick M, McKinlay K, Scott NB. Epidural vs. lumbar plexus infusions following total knee arthroplasty: randomized controlled trial. Eur J Anaesthesiol 2008; 25: 502-7.

16. Macalou D, Trueck S, Meuret P, Heck M, Vial F, Ouologuem S, et al. Postoperative analgesia after total knee replacement: the effect of an obturator nerve block added to the femoral 3-in-1 nerve block. Anesth Analg 2004; 99: 251-4.

17. Kaloul I, Guay J, Côté C, Fallaha M. The posterior lumbar plexus (psoas compartment) block and the three-in-one femoral nerve block provide similar postoperative analgesia after total knee replacement. Can J Anaesth 2004; 51: 45-51.

18. Raimer C, Priem K, Wiese AA, Birnbaum J, Dirkmorfeld LM, Mossner A, et al. Continuous psoas and sciatic block after knee arthroplasty: good effects compared to epidural analgesia or i.v. opioid analgesia: a prospective study of 63 patients. Acta Orthop 2007; 78: 193-200.

19. Watson MW, Mitra D, McLintock TC, Grant SA. Continuous versus single-injection lumbar plexus blocks: comparison of the effects on morphine use and early recovery after total knee arthroplasty. Reg Anesth Pain Med 2005; 30: 541-7.

20. Morin AM, Kratz CD, Eberhart LH, Dinges G, Heider E, Schwarz N, et al. Postoperative analgesia and functional recovery after totalknee replacement: comparison of a continuous posterior lumbar plexus (psoas compartment) block, a continuous femoral nerve block, and the combination of a continuous femoral and sciatic nerve block. Reg Anesth Pain Med 2005; 30: 434-45.

21. Chayen D, Nathan H, Chayen M. The psoas compartment block. Anesthesiology 1976; 45: 95-9. 